\title{
Towards Certified Meta-Programming with Typed Template-CoQ
}

\author{
Abhishek Anand ${ }^{1(\otimes)}$, Simon Boulier ${ }^{2}$, Cyril Cohen $^{3}$, Matthieu Sozeau $^{4}$, \\ and Nicolas Tabareau ${ }^{2}$ \\ 1 Cornell University, Ithaca, NY, USA \\ aa755@cs. cornell.edu \\ 2 Gallinette Project-Team, Inria Nantes, Rennes, France \\ ${ }^{3}$ Université Côte d'Azur, Inria, Nice, France \\ 4 Pi.R2 Project-Team, Inria Paris and IRIF, Paris, France
}

\begin{abstract}
Template-CoQ (https://template-coq.github.io/templatecoq) is a plugin for CoQ, originally implemented by Malecha [18], which provides a reifier for CoQ terms and global declarations, as represented in the CoQ kernel, as well as a denotation command. Initially, it was developed for the purpose of writing functions on CoQ's AST in GALLINA. Recently, it was used in the CERTICOQ certified compiler project [4], as its front-end language, to derive parametricity properties [3], and to extract CoQ terms to a CBV $\lambda$-calculus [13]. However, the syntax lacked semantics, be it typing semantics or operational semantics, which should reflect, as formal specifications in CoQ, the semantics of CoQ's type theory itself. The tool was also rather bare bones, providing only rudimentary quoting and unquoting commands. We generalize it to handle the entire Calculus of Inductive Constructions (CIC), as implemented by CoQ, including the kernel's declaration structures for definitions and inductives, and implement a monad for general manipulation of CoQ's logical environment. We demonstrate how this setup allows CoQ users to define many kinds of general purpose plugins, whose correctness can be readily proved in the system itself, and that can be run efficiently after extraction. We give a few examples of implemented plugins, including a parametricity translation. We also advocate the use of TEMPLATE-CoQ as a foundation for higher-level tools.
\end{abstract}

\section{Introduction}

Meta-programming is the art of writing programs (in a meta-language) that produce or manipulate programs (written in an object language). In the setting of dependent type theory, the expressivity of the language permits to consider the case were the meta and object languages are actually the same, accounting for well-typedness. This idea has been pursued in the work on inductive-recursive (IR) and quotient inductive-inductive types (QIIT) in Agda to reflect a syntactic model of a dependently-typed language within another one [2,9]. These term encodings include type-correctness internally by considering only well-typed terms of the syntax, i.e. derivations. However, the use of IR or QIITs complicates considerably the

(C) The Author(s) 2018

J. Avigad and A. Mahboubi (Eds.): ITP 2018, LNCS 10895, pp. 20-39, 2018.

https://doi.org/10.1007/978-3-319-94821-8_2 
meta-theory of the meta-language which makes it difficult to coincide with the object language represented by an inductive type. More problematically in practice, the concision and encapsulation of the syntactic encoding has the drawback that it is very difficult to use because any function from the syntax can be built only at the price of a proof that it respects typing, conversion or any other features described by the intrinsically typed syntax right away.

Other works have taken advantage of the power of dependent types to do meta-programming in a more progressive manner, by first defining the syntax of terms and types; and then defining out of it the notions of reduction, conversion and typing derivation $[11,26]$ (the introduction of [11] provides a comprehensive review of related work in this area). This can be seen as a type-theoretic version of the functional programming language designs such as TEMPLATE HASKELL [22] or MetaML [24]. This is also the approach taken by Malecha in his thesis [18], where he defined Template-CoQ, a plugin which defines a correspondenceusing quoting and unquoting functions - between CoQ kernel terms and inhabitants of an inductive type representing internally the syntax of the calculus of inductive constructions (CIC), as implemented in CoQ. It becomes thus possible to define programs in CoQ that manipulate the representation of CoQ terms and reify them as functions on CoQ terms. Recently, its use was extended for the needs of the CERTICOQ certified compiler project [4], which uses it as its frontend language. It was also used by Anand and Morissett [3] to formalize a modified parametricity translation, and to extract CoQ terms to a CBV $\lambda$-calculus [13]. All of these translations however lacked any means to talk about the semantics of the reified programs, only syntax was provided by Template-Coq. This is an issue for CERTICOQ for example where both a non-deterministic small step semantics and a deterministic call-by-value big step semantics for CIC terms had to be defined and preserved by the compiler, without an "official" specification to refer to.

This paper proposes to remedy this situation and provides a formal semantics of CoQ's implemented type theory, that can independently be refined and studied. The advantage of having a very concrete untyped description of CoQ terms (as opposed to IR or QIITs definitions) together with an explicit type checker is that the extracted type-checking algorithm gives rise to an OCAML program that can directly be used to type-check CoQ kernel terms. This opens a way to a concrete solution to bootstrap CoQ by implementing the Coq kernel in CoQ. However, a complete reification of CIC terms and a definition of the checker are not enough to provide a meta-programming framework in which CoQ plugins could be implemented. One needs to get access to CoQ logical environments. This is achieved using a monad that reifies CoQ general commands, such as lookups and declarations of constants and inductive types.

As far as we know this is the only reflection framework in a dependentlytyped language allowing such manipulations of terms and datatypes, thanks to the relatively concise representation of terms and inductive families in CIC. Compared to the MetaCoQ project [27], LEan's tactic monad [12], or AGDA's reflection framework [26], our ultimate goal is not to interface with CoQ's 


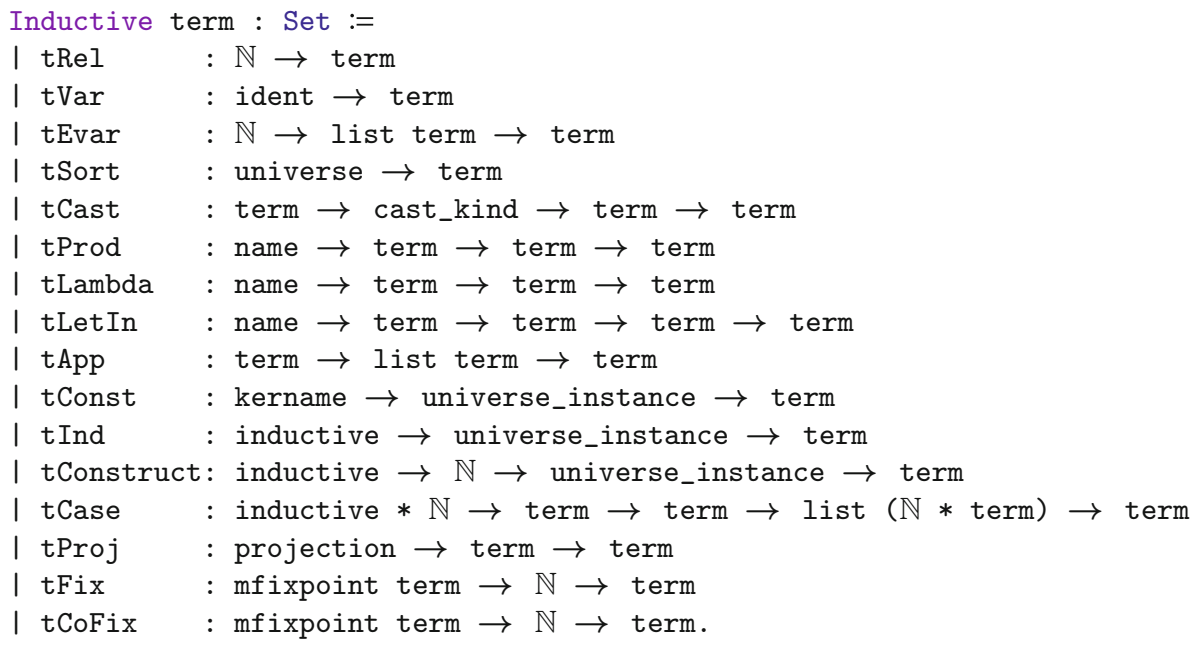

Fig. 1. Representation of the syntax in Template-Coq

unification and type-checking algorithms, but to provide a self-hosted, bootstrappable and verifiable implementation of these algorithms. On one hand, this opens the possibility to verify the kernel's implementation, a problem tackled by BARRAS [6] using set-theoretic models. On the other hand we also advocate for the use of TEMPLATE-COQ as a foundation on which higher-level tools can be built: meta-programs implementing translations, boilerplate-generating tools, domain-specific proof languages, or even general purpose tactic languages.

Plan of the Paper. In Sect. 2, we present the complete reification of CoQ terms, covering the entire CIC and define in Sect. 3 the type-checking algorithm of Coq reified terms in Coq. In Sect. 4, we show the definition of a monad for general manipulation of CoQ's logical environment and use it to define plugins for various translations from CoQ to CoQ (Sect. 5). Finally, we discuss related and future work in Sect. 6.

\section{Reification of CoQ Terms}

Reification of Syntax. The central piece of Template-Coq is the inductive type term which represents the syntax of CoQ terms, as defined in Fig. 1. This inductive follows directly the constr datatype of CoQ terms in the OCAML code of COQ, except for the use of OCAML's native arrays and strings; an upcoming extension of COQ [5] with such features should solve this mismatch.

Constructor tRel represents variables bound by abstractions (introduced by tLambda), dependent products (introduced by tProd) and local definitions (introduced by tLetIn), the natural number is a De Bruijn index. The name is a printing annotation. 
Sorts are represented with tSort, which takes a universe as argument. A universe is the supremum of a (non-empty) list of level expressions, and a level is either Prop, Set, a global level or a De Bruijn polymorphic level variable.

Inductive level := 1Prop | lSet | Level (_ : string) | Var (_ : $\mathbb{N}$ ). Definition universe $:=$ list (level $*$ bool). (* level+1 if true $*$ )

The application (introduced by tApp) is n-ary. The tConst, tInd and tConstruct constructors represent references to constants (definitions or axioms), inductives, or constructors of an inductive type. The universe_instances are non-empty only for polymorphic constants. Finally, tCase represents pattern-matchings, tProj primitive projections, $\mathrm{tFix}$ fixpoints and tCoFix cofixpoints.

Quoting and Unquoting of Terms. TEMPlate-Coq provides a lifting from concrete syntax to reified syntax (quoting) and the converse (unquoting). It can reify and reflect all kernel COQ terms.

The command Quote Definition reifies the syntax of a term. For instance,

Quote Definition $f:=($ fun $x \Rightarrow x+0)$.

generates the term $f$ defined as

$f=$ tLambda (nNamed "x") (tInd $\{\mid$ inductive_mind := "Coq.Init.Datatypes. nat"; inductive_ind $:=0 \mid\}$ [ ] ) (tApp (tConst "Coq.Init.Nat.add" []) [tRel 0 ; tConstruct $\{\mid$ inductive_mind := "Coq. Init.Datatypes.nat"; inductive_ind $\left.:=\begin{array}{lllll}0 & \mid & 0 & {[}\end{array}\right]$ : term

On the converse, the command Make Definition constructs a term from its syntax. This example below defines zero to be 0 of type $\mathbb{N}$.

Make Definition zero := tConstruct (mkInd "Coq.Init.Datatypes.nat" 0) 0 [] .

where mkInd $\mathrm{n} \mathrm{k}$ is the $\mathrm{k}^{\text {th }}$ inductive of the mutual block of the name $\mathrm{n}$.

Reification of Environment. In COQ, the meaning of a term is relative to an environment, which must be reified as well. Environments consist of three parts: (i) a graph of universes (ii) declarations of definitions, axioms and inductives (iii) a local context registering types of De Bruijn indexes.

As we have seen in the syntax of terms, universe levels are not given explicitly in Coq. Instead, level variables are introduced and constraints between them are registered in a graph of universes. This is the way typical ambiguity is implemented in COQ. A constraint is given by two levels and a constraint_type (Lt, Le or Eq):

Definition univ_constraint $:=$ Level $*$ constraint_type $*$ Level.

Then the graph is given by a set of level variables and one of constraints. Sets, coming from the CoQ standard library, are implemented using lists without duplicates. Levelset.t means the type $t$ of the module Levelset.

Definition uGraph $:=$ LevelSet.t $*$ ConstraintSet.t. 
Functions to query the graph are provided, for the moment they rely on a naive implementation of the Bellman-Ford algorithm. check_leq u1 u2 checks if the graph enforces $u 1 \leq \mathrm{u} 2$ and no_universe_inconsistency checks that the graph has no negative cycle.

Constant and inductive declarations are grouped together, properly ordered according to dependencies, in a global context (global_ctx), which is a list of global declarations (global_decl).

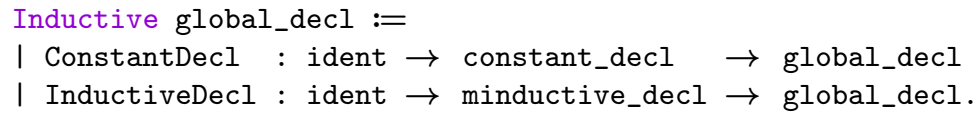

Definitions and axioms just associate a name to a universe context, and two terms for the optional body and type. Inductives are more involved:

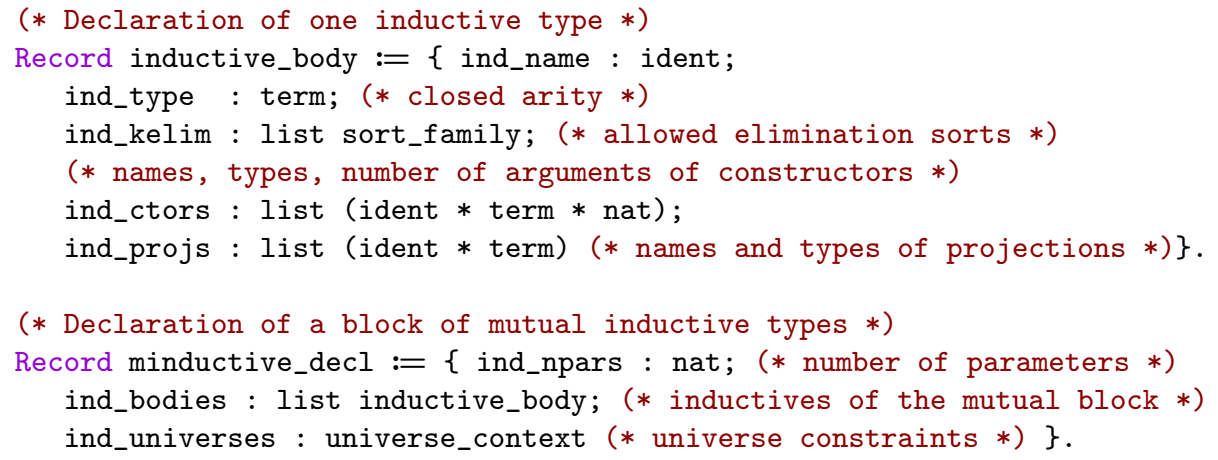

In CoQ internals, there are in fact two ways of representing a declaration: either as a "declaration" or as an "entry". The kernel takes entries as input, type-check them and elaborate them to declarations. In Template-Coq, we provide both, and provide an erasing function mind_decl_to_entry from declarations to entries for inductive types.

Finally, local contexts are just list of local declarations: a type for lambda bindings and a type and a body for let bindings.

Quoting and Unquoting the Environment. Template-CoQ provides the command Quote Recursively Definition to quote an environment. This command crawls the environment and quote all declarations needed to typecheck a given term.

The other way, the commands Make Inductive allows declaring an inductive type from its entry. For instance the following redefines a copy of $\mathbb{N}$ :

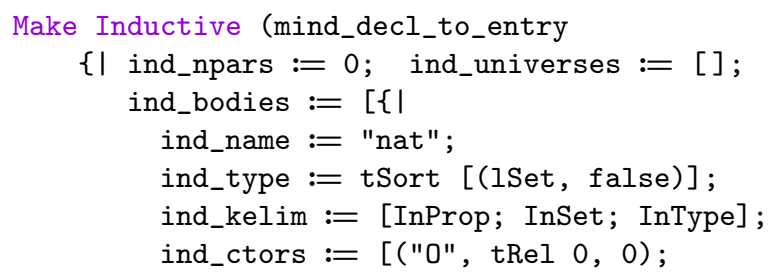




$$
\begin{aligned}
& \text { ("S", tProd nAnon (tRel 0) (tRel 1), 1)]; } \\
& \text { ind_projs }:=\left[\begin{array}{ll|}
] & \mid\}
\end{array}\right] \text { |\}). }
\end{aligned}
$$

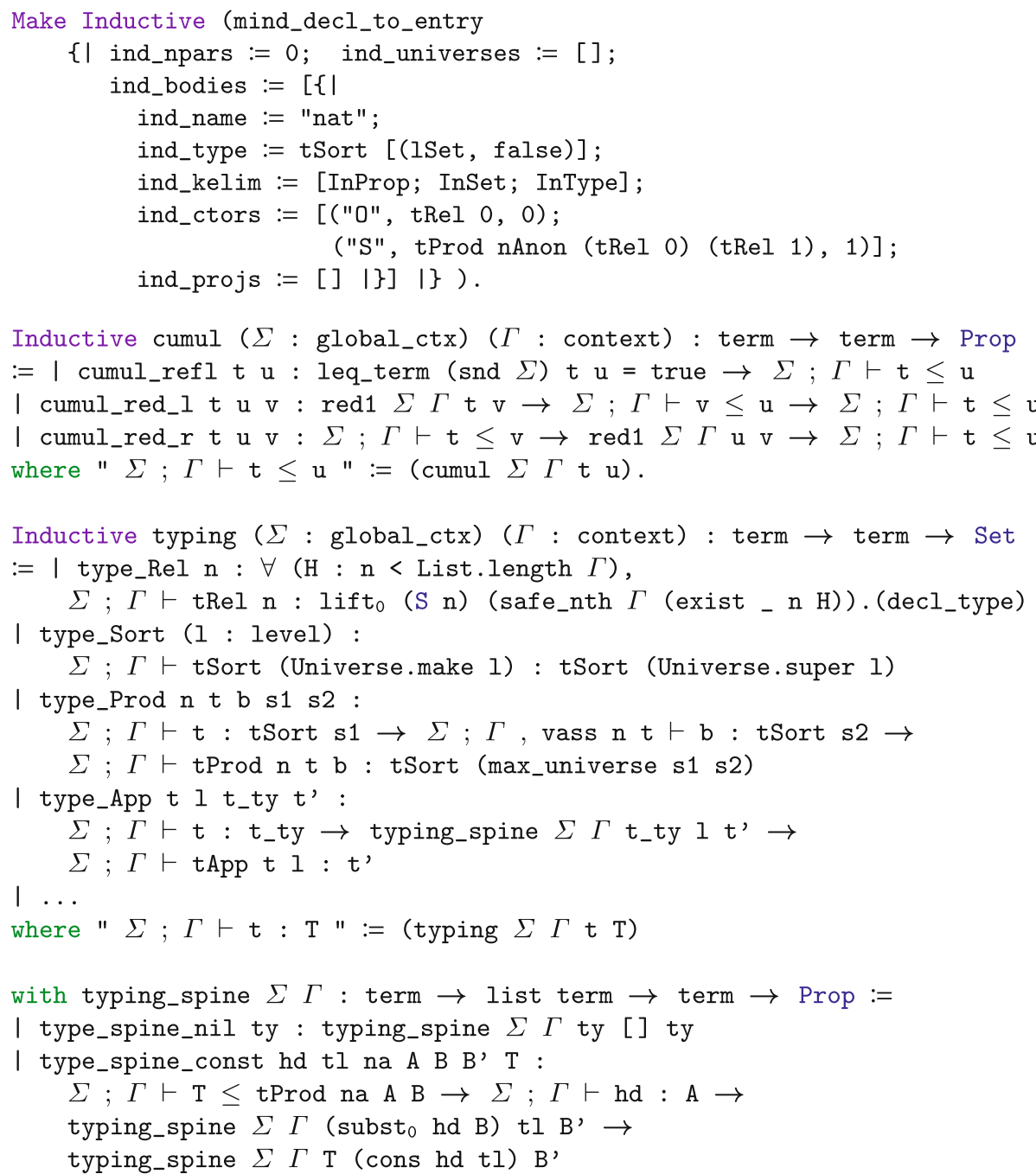

Fig. 2. Typing judgment for terms, excerpt

More examples of use of quoting/unquoting commands can be found in the file test-suite/demo.v. 


\section{Type Checking CoQ in CoQ}

In Fig. 2, we present (an excerpt of) the specification of the typing judgment of the kernel of COQ using the inductive type typing. It represents all the typing rules of $\mathrm{COQ}^{1}$. This includes the basic dependent lambda calculus with lets, global references to inductives and constants, the match construct and primitive projections. Universe polymorphic definitions and the well-formedness judgment for global declarations are dealt with as well.

The only ingredients missing are the guard check for fixpoint and productivity of cofixpoints and the positivity condition of mutual (co-) inductive types. They are work-in-progress.

The typing judgment typing is mutually defined with typing_spine to account for n-ary applications. Untyped reduction red1 and cumulativity cumul can be defined separately.

Implementation. To test this specification, we have implemented the basic algorithms for type-checking in COQ, that is, we implement type inference: given a context and a term, output its type or produce a type error. All the rules of type inference are straightforward except for cumulativity. The cumulativity test is implemented by comparing head normal forms for a fast-path failure and potentially calling itself recursively, unfolding definitions at the head in CoQ's kernel in case the heads are equal. We implemented weak-head reduction by mimicking CoQ's kernel implementation, which is based on an abstract machine inspired by the KAM. COQ's machine optionally implements a variant of lazy, memoizing evaluation (which can have mixed results, see CoQ's PR \#555 for example), that feature has not been implemented yet.

The main difference with the OCAML implementation is that all of the functions are required to be shown terminating in Coq. One possibility could be to prove the termination of type-checking separately but this amounts to prove in particular the normalization of CIC which is a complex task. Instead, we simply add a fuel parameter to make them syntactically recursive and make Out0fFuel a type error, $i$.e., we are working in a variant of the option monad.

Bootstrapping It. We can extract this checker to OCAML and reuse the setup described in Sect. 2 to connect it with the reifier and easily derive a (partialy verified) alternative checker for CoQ's . vo object files. Our plugin provides a new command Template Check for typechecking definitions using the alternative checker, that can be used as follows:

Require Import Template.TemplateCoqChecker List. Import ListNotations.

Definition foo := List.map (fun $\mathrm{x} \Rightarrow \mathrm{x}+3$ ) [0; 1] .

Template Check foo.

Our initial tests indicate that its running time is comparable to the coqchk checker of CoQ, as expected.

\footnotetext{
${ }^{1}$ We do not treat metavariables which are absent from kernel terms and require a separate environment for their declarations.
} 


\section{Reification of CoQ Commands}

CoQ plugins need to interact with the environment, for example by repeatedly looking up definitions by name, declaring new constants using fresh names, or performing computations. It is desirable to allow such programs to be written in Coq (Gallina) because of the two following advantages. Plugin-writers no longer need to understand the OCAML implementation of COQ and plugins are no longer sensitive to changes made in the OCAML implementation. Also, when plugins implementing syntactic models are proven correct in COQ, they provide a mechanism to add axioms to COQ without compromising consistency (Sect. 5.3).

In general, interactions with the environment have side effects, e.g. the declaration of new constants, which must be described in CoQ's pure setting. To overcome this difficulty, we use the standard "free" monadic setting to represent the operations involved in interacting with the environment, as done for instance in Mtac [27].

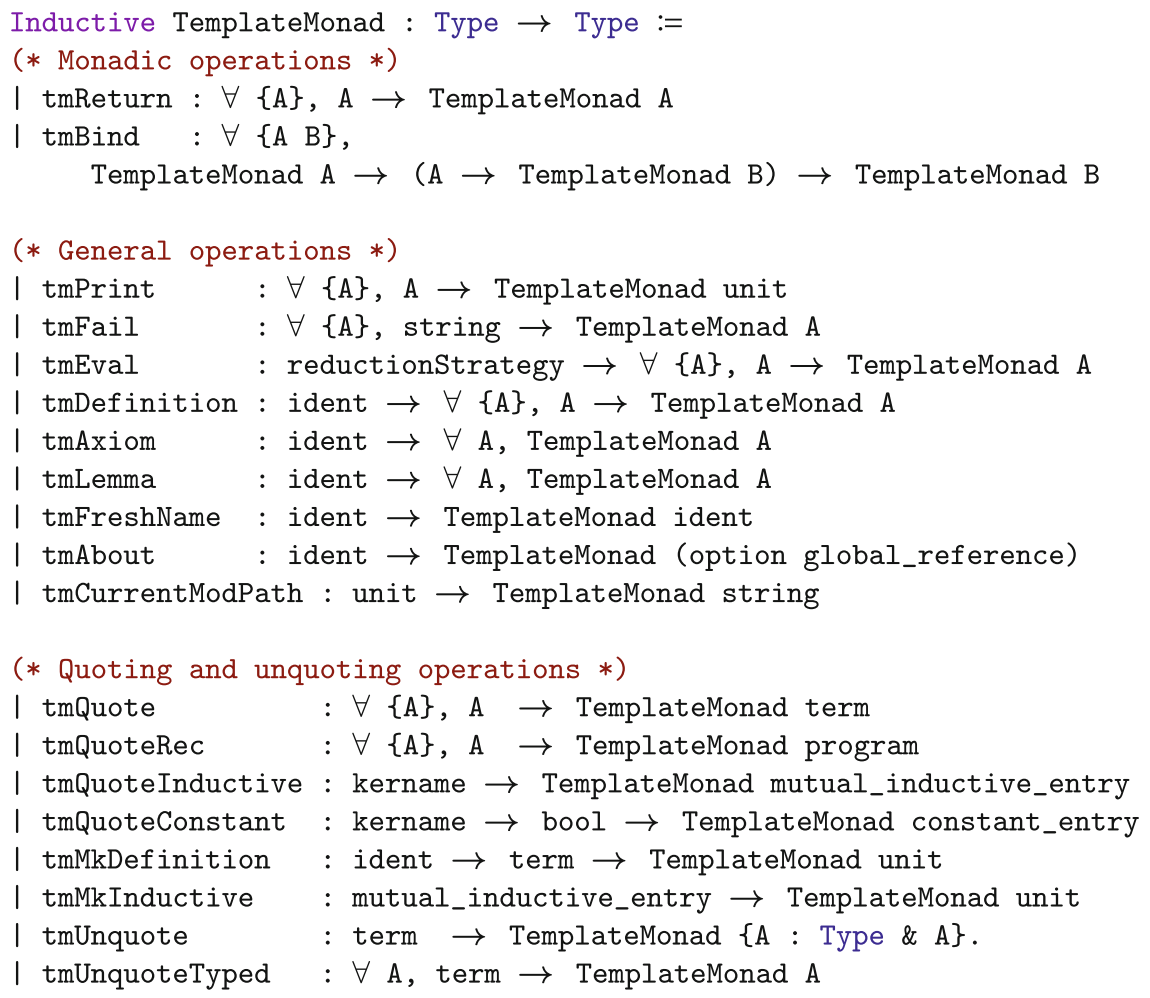

Fig. 3. The monad of commands 
Table 1. Main Template-Coq commands

\begin{tabular}{|c|c|c|}
\hline Vernacular command & $\begin{array}{l}\text { Reified command with } \\
\text { its arguments }\end{array}$ & Description \\
\hline Eval & tmEval red $t$ & $\begin{array}{l}\text { Returns the evaluation of } t \\
\text { following the evaluation strategy } \\
\text { red (cbv, cbn, hnf, all or lazy) }\end{array}$ \\
\hline Definition & tmDefinition id $t$ & $\begin{array}{l}\text { Makes the definition id }:=t \\
\text { and returns the created constant } \\
\text { id }\end{array}$ \\
\hline Axiom & tmAxiom id $\mathrm{A}$ & $\begin{array}{l}\text { Adds the axiom id of type } A \text { and } \\
\text { returns the created constant id }\end{array}$ \\
\hline Lemma & tmLemma id $\mathrm{A}$ & $\begin{array}{l}\text { Generates an obligation of type } \\
\text { A, returns the created constant } \\
\text { id after all obligations close }\end{array}$ \\
\hline \multirow[t]{5}{*}{ About or Locate } & tmAbout id & $\begin{array}{l}\text { Returns Some gr if id is a } \\
\text { constant in the current } \\
\text { environment and gr is the } \\
\text { corresponding global reference. } \\
\text { Returns None otherwise. }\end{array}$ \\
\hline & tmQuote $t$ & $\begin{array}{l}\text { Returns the syntax of } t \text { (of type } \\
\text { term) }\end{array}$ \\
\hline & tmQuoteRec $t$ & $\begin{array}{l}\text { Returns the syntax of } t \text { and all } \\
\text { the declarations on which it } \\
\text { depends }\end{array}$ \\
\hline & tmQuoteInductive $\mathrm{kn}$ & $\begin{array}{l}\text { Returns the declaration of the } \\
\text { inductive } \mathrm{kn}\end{array}$ \\
\hline & tmQuoteConstant $\mathrm{kn} \mathrm{b}$ & $\begin{array}{l}\text { Returns the declaration of the } \\
\text { constant } \mathrm{kn} \text {, if } \mathrm{b} \text { is true the } \\
\text { implementation bypass opacity } \\
\text { to get the body of the constant }\end{array}$ \\
\hline Make Definition & tmMkDefinition id tm & $\begin{array}{l}\text { Adds the definition id }:=t \\
\text { where } t \text { is denoted by } t m\end{array}$ \\
\hline \multirow[t]{3}{*}{ Make Inductive } & tmMkInductive $\mathrm{d}$ & $\begin{array}{l}\text { Declares the inductive denoted } \\
\text { by the declaration } d\end{array}$ \\
\hline & tmUnquote $\mathrm{tm}$ & $\begin{array}{l}\text { Returns the pair }(A ; t) \text { where } t \\
\text { is the term whose syntax is } t m \\
\text { and A it's type }\end{array}$ \\
\hline & tmUnquoteTyped A tm & $\begin{array}{l}\text { Returns the term whose syntax } \\
\text { is tm and checks that it is indeed } \\
\text { of type } \mathrm{A}\end{array}$ \\
\hline
\end{tabular}

TemplateMonad is an inductive family (Fig. 3) such that TemplateMonad A represents a program which will finally output a term of type A. There are special con- 
structor tmReturn and tmBind to provide (freely) the basic monadic operations. We use the monadic syntactic sugar $x \leftarrow t$; $u$ for tmBind $t$ ( $f$ un $x \Rightarrow u$ ).

The other operations of the monad can be classified in two categories: the traditional COQ operations (tmDefinition to declare a new definition, ....) and the quoting and unquoting operations to move between CoQ term and their syntax or to work directly on the syntax (tmMkInductive to declare a new inductive from its syntax for instance). An overview is given in Table 1.

A program prog of type TemplateMonad A can be executed with the command Run TemplateProgram prog. This command is thus an interpreter for TemplateMonadprograms, implemented in OCAML as a traditional CoQ plugin. The term produced by the program is discarded but, and it is the point, a program can have many side effects like declaring a new definition or a new inductive type, printing something, ....

Let's look at some examples. The following program adds the definitions foo $:=12$ and bar $:=$ foo +1 to the current context.

Run TemplateProgram (foo $\leftarrow$ tmDefinition "foo" 12 ; tmDefinition "bar" $($ foo +1$))$.

The program below asks the user to provide an inhabitant of $\mathbb{N}$ (here we provide $3 * 3$ ) and records it in the lemma foo ; prints its normal form ; and records the syntax of its normal form in foo_nf_syntax (hence of type term). We use Program's obligation mechanism ${ }^{2}$ to ask for missing proofs, running the rest of the program when the user finishes providing it. This enables the implementation of interactive plugins.

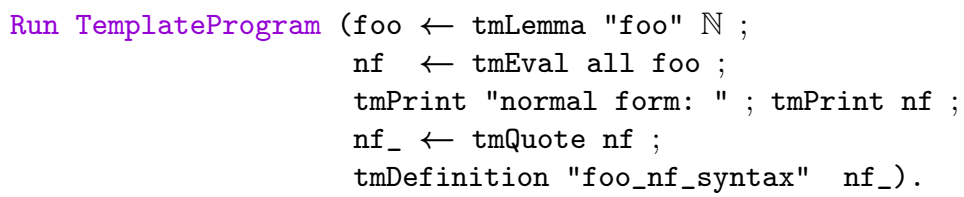

\section{$5 \quad$ Writing Coq Plugins in CoQ}

The reification of syntax, typing and commands of COQ allow writing a CoQ plugin directly inside CoQ, without requiring another language like OCAML and an external compilation phase.

In this section, we describe three examples of such plugins: (i) a plugin that adds a constructor to an inductive type, (ii) a re-implementation of LASSON's parametricity plugin ${ }^{3}$, and (iii) an implementation of a plugin that provides an extension of CIC - using a syntactic translation - in which it is possible to prove the negation of functional extensionality [8].

\footnotetext{
${ }^{2}$ In CoQ, a proof obligation is a goal which has to be solved to complete a definition. Obligations were introduced by Sozeau [23] in the Program mode.

${ }^{3}$ https://github.com/parametricity-coq/paramcoq.
} 


\subsection{A Plugin to Add a Constructor}

Our first example is a toy example to show the methodology of writing plugins in Template-Coq. Given an inductive type I, we want to declare a new inductive type I' which corresponds to I plus one more constructor.

For instance, let's say we have a syntax for lambda calculus:

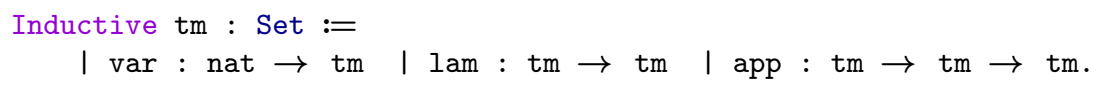

And that in some part of our development, we want to consider a variation of tm with a new constructor, e.g., let in. Then we declare tm' with the plugin by:

Run TemplateProgram

(add_constructor $\mathrm{tm}$ "letin" (fun $\mathrm{tm}$ ' $\Rightarrow \mathrm{tm}$ ' $\rightarrow \mathrm{tm}$ ' $\left.\rightarrow \mathrm{tm} \mathrm{m}^{\prime}\right)$ ).

This command has the same effect as declaring the inductive tm' by hand:

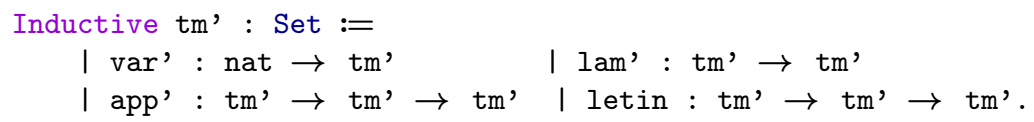

but with the benefit that if tm is changed, for instance by adding one new constructor, then tm' is automatically changed accordingly. We provide other examples in the file test-suite/add_constructor.v, e.g. with mutual inductives.

We will see that it is fairly easy to define this plugin using TEMPLATE-CoQ. The main function is add_constructor which takes an inductive type ind (whose type is not necessarily Type if it is an inductive family), a name idc for the new constructor and the type ctor of the new constructor, abstracted with respect to the new inductive.

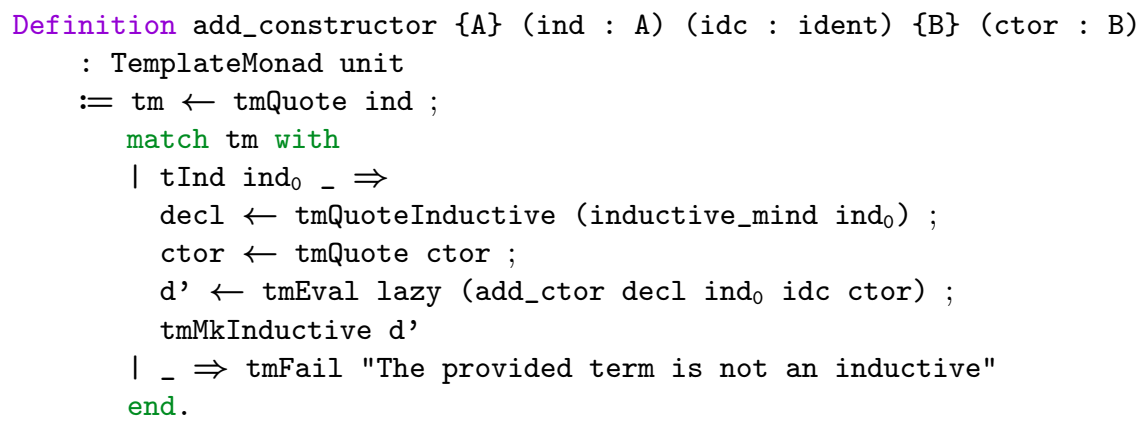

It works in the following way. First the inductive type ind is quoted, the obtained term tm is expected to be a tInd constructor otherwise the function fails. Then the declaration of this inductive is obtained by calling tmQuoteInductive, the constructor is reified too, and an auxiliary function is called to add the constructor to the declaration. After evaluation, the new inductive type is added to the current context with tmMkInductive.

It remains to define the add_ctor auxiliary function to complete the definition of the plugin. It takes a minductive_decl which is the declaration of a block of mutual inductive types and returns a minductive_decl. 


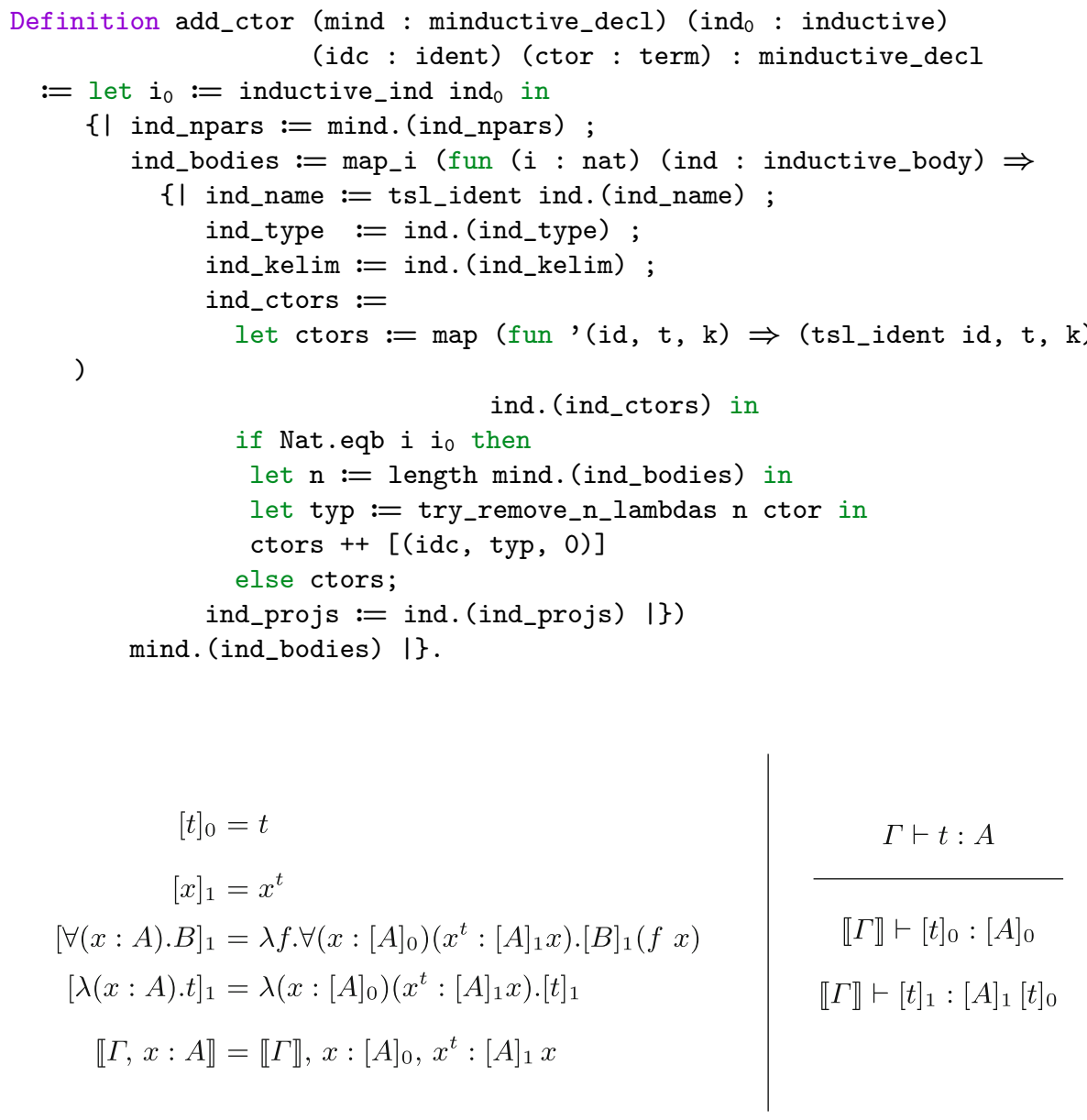

Fig. 4. Unary parametricity translation and soundness theorem, excerpt (from [7])

The declaration of the block of mutual inductive types is a record. The field ind_bodies contains the list of declarations of each inductive of the block. We see that most of the fields of the records are propagated, except for the names which are translated to add some primes and ind_ctors, the list of types of constructors, for which, in the case of the relevant inductive ( $i_{0}$ is its number), the new constructor is added.

\subsection{Parametricity Plugin}

We now show how TEMPLATE-COQ permits to define a parametricity plugin that computes the translation of a term following Reynolds' parametricity [21,25]. We follow the already known approaches of parametricity for dependent type theories [7,15], and provide an alternative to Keller and Lasson's plugin. 
The definition in the unary case is described in Fig. 4. The soundness theorem ensures that, for a term $t$ of type $A,[t]_{1}$ computes a proof of parametricity of $[t]_{0}$ in the sense that it has type $[A]_{1}[t]_{0}$. The definition of the plugin goes in two steps: first the definition of the translation on the syntax of term in TEMPLATECOQ and then the instrumentation to connect it with terms of CoQ using the TemplateMonad. It can be found in the file translations/tsl_param.v.

The parametricity translation of Fig. 4 is total and syntax directed, the two components of the translation [ $]_{0}$ and [ $]_{1}$ are implemented by two recursive functions tsl_paramo and tsl_param . $_{1}$

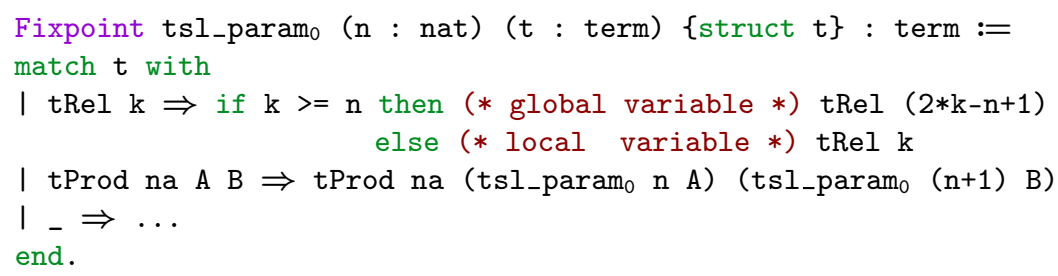

On Fig. 4, the translation is presented in a named setting, so the introduction of new variables does not change references to existing ones. That's why, [ ] 0 is the identity. In the De Bruijn setting of Template-CoQ, the translation has to take into account the shift induced by the duplication of the context. Therefore, the implementation $t s l_{-}$param $_{0}$ of []$_{0}$ is not the identity anymore. The argument $\mathrm{n}$ of $\mathrm{ts} \mathbf{l}_{\text {_p }}$ paramo represents the De Bruijn level from which the variables

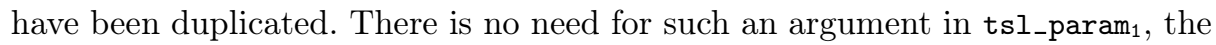
implementation of []$_{1}$, because in this function all variables are duplicated.

The parametricity plugin not only has to be defined on terms of CIC but also on additional terms dealing with the global context. In particular, constants are translated using a translation table which records the translations of previously processed constants. 
Definition tsl_table $:=$ list (global_reference $*$ term).

If a constant is not in the translation table we return a dummy default_term, considered as an error (this could also be handled by an option monad).

We have also implemented the translation of inductives and pattern matching. For instance the translation of the equality type eq produces the inductive type:

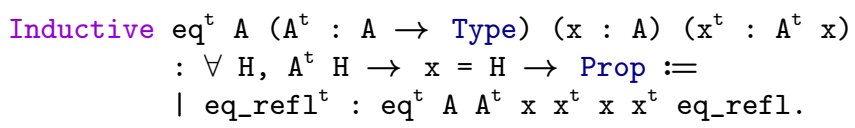

Then $[e q]_{1}$ is given by eq ${ }^{t}$ and [eq_refl $]_{1}$ by eq_refl ${ }^{t}$.

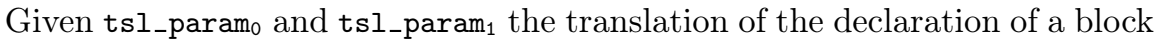
of mutual inductive types is not so hard to get. Indeed, such a declaration mainly consists of the arities of the inductives and the types of constructors; and the one of the translated inductive are produced by translation of the original ones.

Definition tsl_mind_decl (E : tsl_table) (kn : kername)

(mind : minductive_decl) : minductive_decl.

In a similar manner, we can translate pattern-matching. Note that the plugin does not support fixpoints and cofixpoints for the moment.

Now, it remains to connect this translation defined on reified syntax term to terms of CoQ. For this, we define the new command tTranslate in the TemplateMonad.

Definition tTranslate (E : tsl_table) (id : ident)

: TemplateMonad tsl_table.

When id is a definition, the command recovers the body of id (as a term) using tmQuoteConstant and then translates it and records it in a new definition $i^{t}$. The command returns the translation table $E$ extended by (id, $i d^{t}$ ). In the case id is an inductive type or a constructor then the command does basically the same but extends the translation table with both the inductive and the constructors. If $i d$ is an axiom or not a constant the command fails.

Here is an illustration coming from the work of Lasson [16] on the automatic proofs of $(\omega$-)groupoid laws using parametricity. We show that all function of type ID $:=\forall \mathrm{Ax}, \mathrm{x}=\mathrm{y} \rightarrow \mathrm{x}=\mathrm{y}$ are identity functions. First we need to record the translations of eq and ID in a term table of type tsl_table.

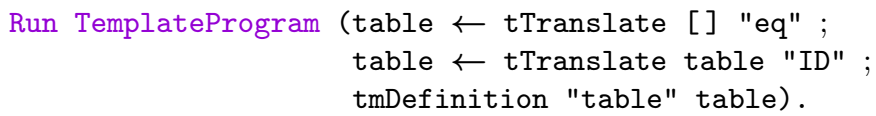

Then we show that every parametric function on ID is pointwise equal to the identity using the predicate fun $\mathrm{y} \Rightarrow \mathrm{x}=\mathrm{y}$.

Lemma param_ID ( $f:$ ID) $: I^{t} f \rightarrow \forall A x$ y $p$, f A x y p $=p$.

Proof.

intros $\mathrm{H} A \mathrm{x}$ y $\mathrm{p}$. destruct $\mathrm{p}$.

destruct ( $\mathrm{H} A$ (fun $\mathrm{y} \Rightarrow \mathrm{x}=\mathrm{y}$ ) $\mathrm{x}$ eq_refl 
$\mathrm{x}$ eq_refl eq_refl (eq_refl ${ }^{\mathrm{t}}{ }_{-}$)) )

reflexivity.

Qed.

Then we define a function myf $:=p \mapsto p \cdot p^{-1} \cdot p$ and get its parametricity proof using the plugin.

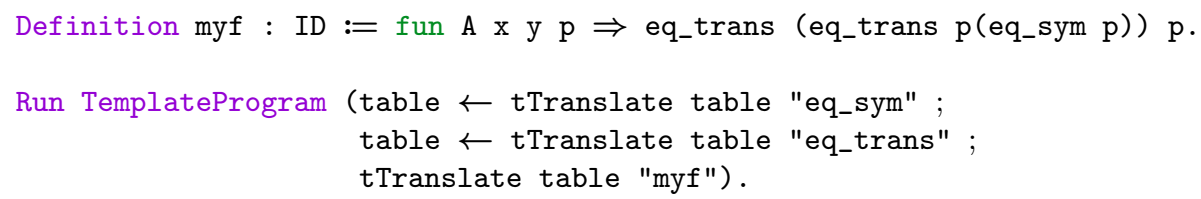

It is then possible to deduce automatically that $p \cdot p^{-1} \cdot p=p$ for all $p: x=y$. Definition free_thm_myf: $\forall$ A x y p, myf A x y $p=p:=$ param_ID myf myf ${ }^{t}$.

\subsection{Intensional Function Plugin}

Our last illustration is a plugin that provides an intensional flavour to functions and thus allows negating functional extensionality (FunExt). This is a simple example of syntactical translation which enriches the logical power of CoQ, in the sense that new theorems can be proven (as opposed to the parametricity translation which is conservative over CIC). See [8] for an introduction to syntactical translations and a complete description of the intensional function translation.

$$
\begin{array}{rlrl}
{[x]} & :=x & {[\lambda(x: A) \cdot t]} & :=(\lambda(x:[A]) \cdot[t], \text { true }) \\
{[t u]:=\left(\pi_{1}[t]\right)[u]} & {[\forall(x: A) \cdot B]:} & =(\forall(x:[A]) \cdot[B]) \times \mathbb{B}
\end{array}
$$

Fig. 5. Intensional function translation, excerpt (from [8])

Even if the translation is very simple as it just adds a boolean to every function (Fig. 5), this time, it is not fully syntax directed. Indeed the notation for pairs hide some types:

$$
[f u n(x: A) \Rightarrow t]:=\operatorname{pair}(\forall x:[A] \text {. ?T) bool (fun }(x:[A]) \Rightarrow[t]) \text { true }
$$

and we can not recover the type ?T from the source term. There is thus a mismatch between the lambdas which are not fully annotated and the pairs which are. $^{4}$

However we can use the type inference algorithm of Sect. 3 implemented on TEmPlate-CoQ terms to recover the missing information.

$$
\begin{aligned}
{[\text { fun }(\mathrm{x}: \mathrm{A}) \Rightarrow \mathrm{t}]:=} & \operatorname{let} \mathrm{B}:=\operatorname{infer} \Sigma(\Gamma, \mathrm{x}:[\mathrm{A}]) \mathrm{t} \text { in } \\
& \text { pair }(\forall(\mathrm{x}:[\mathrm{A}]) . \mathrm{B}) \text { bool (fun }(\mathrm{x}:[\mathrm{A}]) \Rightarrow[\mathrm{t}]) \text { true }
\end{aligned}
$$

${ }^{4}$ Note that there is a similar issue with applications and projections, but which can be circumvented this time using (untyped) primitive projections. 
Compared to the parametricity plugin, the translation function has a more complex type as it requires the global and local contexts. However, we can generalize the tTranslate command so that it can be used for both the parametricity and the intensional function plugins. The implementation is in the files translations/translation_utils.v and translations/tsl_fun.v.

Extending CoQ Using Plugins. The intensional translation extends the logical power of $\mathrm{CoQ}$ as it is possible for instance to negate FunExt. In this perspective, we defined a new command:

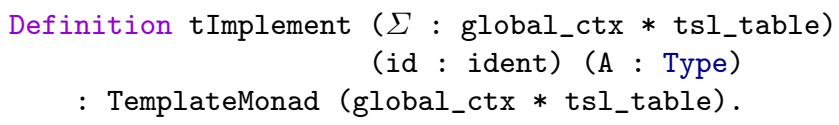

which computes the translation A' of A, then asks the user to inhabit the type A' by generating a proof obligation and then safely adds the axiom id of type A to the current context. By safely, we mean that the correction of the translation ensures that no inconsistencies are introduced.

For instance, here is how to negate FunExt. We use for that two pairs ( $f$ un $x \Rightarrow x$; true) and ( $f$ un $x \Rightarrow x$; false) in the interpretation of functions from unit to unit, which are extensionally both the identity, but differ intensionally on their boolean.

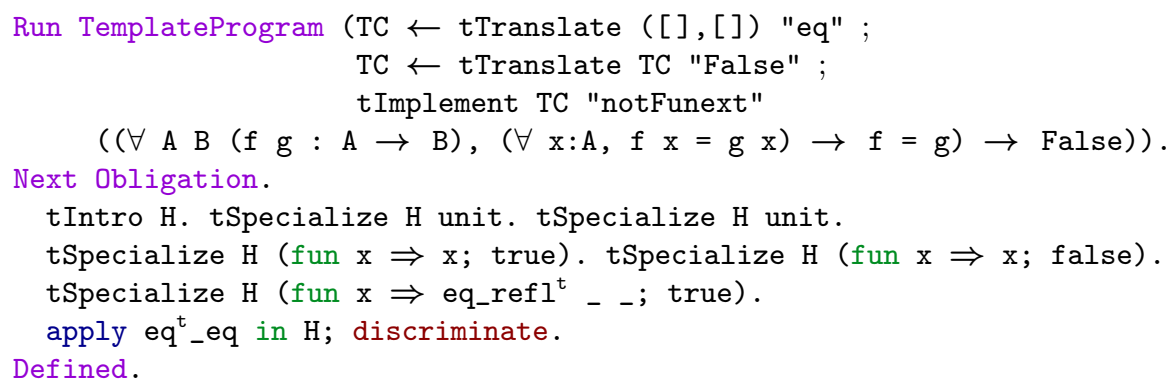

where tIntro and tSpecialize are special versions of the corresponding intro and specialize tactics of CoQ to deal with extra booleans appearing in the translated terms. After this command, the axiom notFunext belongs to the environment, as if it where added with the Axiom command. But as we have inhabited the translation of its type, the correctness of the translation ensures that no inconsistency were introduced.

Note that we could also define another translation, e.g. the setoid translation, in which FunExt is inhabited. This is not contradictory as the two translations induce two different logical extensions of CoQ, which can not be combined. 


\section{Related Work and Future Work}

Meta-Programming is a whole field of research in the programming languages community, we will not attempt to give a detailed review of related work here. In contrast to most work on meta-programming, we provide a very rough interface to the object language: one can easily build ill-scoped and ill-typed terms in our framework, and staging is basic. However, with typing derivations we provide a way to verify meta-programs and ensure that they do make sense.

The closest cousin of our work is the Typed Syntactic Meta-Programming [11] proposal in AGDA, which provides a well-scoped and well-typed interface to a denotation function, that can be used to implement tactics by reflection. We could also implement such an interface, asking for a proof of well-typedness on top of the tmUnquoteTyped primitive of our monad.

Intrinsically typed representations of terms in dependent type-theory is an area of active research. Most solutions are based on extensions of Martin-Löf Intensional Type Theory with inductive-recursive or quotient inductive-inductive types [2,9], therefore extending the meta-theory. Recent work on verifying soundness and completeness of the conversion algorithm of a dependent type theory (with natural numbers, dependent products and a universe) in a type theory with IR types [1] gives us hope that this path can nonetheless be taken to provide the strongest guarantees on our conversion algorithm. The intrinsically-typed syntax used there is quite close to our typing derivations.

Another direction is taken by the (Euf certified compiler [19], which restricts itself to a fragment of COQ for which a total denotation function can be defined, in the tradition of definitional interpreters advocated by Chlipala [10]. This setup should be readily accommodated by TEMPLATE-COQ.

The translation + plugin technique paves the way for certified translations and the last piece will be to prove correctness of such translations. By correctness we mean computational soundness and typing soundness (see [8]), and both can be stated in Template-Coq. Anand has made substantial attempts in this direction to prove the computational soundness, in TEMPLATE-CoQ, of a variant of parametricity providing stronger theorems for free on propositions [3]. This included as a first step a move to named syntax that could be reused in other translations.

Our long term goal is to leverage this technique to extend the logical and computational power of CoQ using, for instance, the forcing translation [14] or the weaning translation [20].

When performance matters, we can extract the translation to OCAML and use it like any ordinary CoQ plugin. This relies on the correctness of extraction, but in the untyped syntax + typing judgment setting, extraction of translations is almost an identity pretty-printing phase, so we do not lose much confidence. We can also implement a template monad runner in OCAML to run the plugins outside CoQ. Our first experiments show that we could gain a factor 10 for the time needed to compute the translation of a term. Another solution would be to use the certified CERTICOQ compiler, once it supports a kind of foreign function interface, to implement the TemplateMonad evaluation. 
The last direction of extension is to build higher-level tools on top of the syntax: the unification algorithm described in [28] is our first candidate. Once unification is implemented, we can look at even higher-level tools: elaboration from concrete syntax trees, unification hints like canonical structures and type class resolution, domain-specific and general purpose tactic languages. A key inspiration in this regard is the work of Malecha and Bengston [17] which implemented this idea on a restricted fragment of CIC.

Acknowledgments. This work is supported by the CoqHoTT ERC Grant 64399 and the NSF grants CCF-1407794, CCF-1521602, and CCF-1646417.

\section{References}

1. Abel, A., Öhman, J., Vezzosi, A.: Decidability of conversion for type theory in type theory. PACMPL 2(POPL), 23:1-23:29 (2018). http://doi.acm.org/10.1145/ 3158111

2. Altenkirch, T., Kaposi, A.: Type theory in type theory using quotient inductive types. In: POPL 2016, pp. 18-29. ACM, New York (2016). http://doi.acm.org/10. $1145 / 2837614.2837638$

3. Anand, A., Morrisett, G.: Revisiting parametricity: inductives and uniformity of propositions. In: CoqPL 2018, Los Angeles, CA, USA (2018)

4. Anand, A., Appel, A., Morrisett, G., Paraskevopoulou, Z., Pollack, R.,Belanger, O.S., Sozeau, M., Weaver, M.: CertiCoq: a verified compiler for Coq. In: CoqPL, Paris, France (2017). http://conf.researchr.org/event/CoqPL-2017/main-certicoqa-verified-compiler-for-coq

5. Armand, M., Grégoire, B., Spiwack, A., Théry, L.: Extending CoQ with imperative features and its application to SAT verification. In: Kaufmann, M., Paulson, L.C. (eds.) ITP 2010. LNCS, vol. 6172, pp. 83-98. Springer, Heidelberg (2010). https:// doi.org/10.1007/978-3-642-14052-5_8

6. Barras, B.: Auto-validation d'un système de preuves avec familles inductives. Thèse de doctorat, Université Paris 7, November 1999

7. Bernardy, J.P., Jansson, P., Paterson, R.: Proofs for free: parametricity for dependent types. J. Funct. Program. 22(2), 107-152 (2012)

8. Boulier, S., Pédrot, P.M., Tabareau, N.: The next 700 syntactical models of type theory. In: CPP 2017, pp. 182-194. ACM, Paris (2017)

9. Chapman, J.: Type theory should eat itself. Electron. Notes Theor. Comput. Sci. 228, 21-36 (2009). Proceedings of LFMTP 2008. http://www.sciencedirect.com/ science/article/pii/S157106610800577X

10. Chlipala, A.: Certified Programming with Dependent Types, vol. 20. MIT Press, Cambridge (2011)

11. Devriese, D., Piessens, F.: Typed syntactic meta-programming. In: ICFP 2013, vol. 48, pp. 73-86. ACM (2013). http://doi.acm.org/10.1145/2500365.2500575

12. Ebner, G., Ullrich, S., Roesch, J., Avigad, J., de Moura, L.: A metaprogramming framework for formal verification, pp. 34:1-34:29, September 2017

13. Forster, Y., Kunze, F.: Verified extraction from Coq to a lambda-calculus. In: Coq Workshop 2016 (2016). https://www.ps.uni-saarland.de/forster/coq-workshop$16 /$ abstract-coq-ws- 16 .pdf 
14. Jaber, G., Lewertowski, G., Pédrot, P.M., Sozeau, M., Tabareau, N.: The definitional side of the forcing. In: LICS 2016, New York, NY, USA, pp. 367-376 (2016). http://doi.acm.org/10.1145/2933575.2935320

15. Keller, C., Lasson, M.: Parametricity in an impredicative sort. CoRR abs/1209.6336 (2012). http://arxiv.org/abs/1209.6336

16. Lasson, M.: Canonicity of weak $\omega$-groupoid laws using parametricity theory. Electron. Notes Theor. Comput. Sci. 308, 229-244 (2014)

17. Malecha, G., Bengtson, J.: Extensible and efficient automation through reflective tactics. In: Thiemann, P. (ed.) ESOP 2016. LNCS, vol. 9632, pp. 532-559. Springer, Heidelberg (2016). https://doi.org/10.1007/978-3-662-49498-1_21

18. Malecha, G.M.: Extensible proof engineering in intensional type theory. Ph.D. thesis, Harvard University (2014)

19. Mullen, E., Pernsteiner, S., Wilcox, J.R., Tatlock, Z., Grossman, D.: (Euf: minimizing the Coq extraction TCB. In: Proceedings of CPP 2018, pp. 172-185 (2018). http://doi.acm.org/10.1145/3167089

20. Pédrot, P., Tabareau, N.: An effectful way to eliminate addiction to dependence. In: LICS 2017, Reykjavik, Iceland, pp. 1-12 (2017). https://doi.org/10.1109/LICS. 2017.8005113

21. Reynolds, J.C.: Types, abstraction and parametric polymorphism. In: IFIP Congress, pp. 513-523 (1983)

22. Sheard, T., Jones, S.P.: Template meta-programming for Haskell. SIGPLAN Not. 37(12), 60-75 (2002). http://doi.acm.org/10.1145/636517.636528

23. Sozeau, M.: Programming finger trees in Coq. In: ICFP 2007, pp. 13-24. ACM, New York (2007). http://doi.acm.org/10.1145/1291151.1291156

24. Taha, W., Sheard, T.: Multi-stage programming with explicit annotations. In: PEPM 1997, pp. 203-217. ACM, New York (1997). http://doi.acm.org/10.1145/ 258993.259019

25. Wadler, P.: Theorems for free! In: Functional Programming Languages and Computer Architecture, pp. 347-359. ACM Press (1989)

26. van der Walt, P., Swierstra, W.: Engineering proof by reflection in Agda. In: Hinze, R. (ed.) IFL 2012. LNCS, vol. 8241, pp. 157-173. Springer, Heidelberg (2013). https://doi.org/10.1007/978-3-642-41582-1_10

27. Ziliani, B., Dreyer, D., Krishnaswami, N.R., Nanevski, A., Vafeiadis, V.: Mtac: a monad for typed tactic programming in Coq. J. Funct. Program. 25 (2015). https://doi.org/10.1017/S0956796815000118

28. Ziliani, B., Sozeau, M.: A comprehensible guide to a new unifier for CIC including universe polymorphism and overloading. J. Funct. Program. 27, e10 (2017). http://www.irif.univ-paris-diderot.fr/sozeau/research/publications/ drafts/unification-jfp.pdf 
Open Access This chapter is licensed under the terms of the Creative Commons Attribution 4.0 International License (http://creativecommons.org/licenses/by/4.0/), which permits use, sharing, adaptation, distribution and reproduction in any medium or format, as long as you give appropriate credit to the original author(s) and the source, provide a link to the Creative Commons license and indicate if changes were made.

The images or other third party material in this chapter are included in the chapter's Creative Commons license, unless indicated otherwise in a credit line to the material. If material is not included in the chapter's Creative Commons license and your intended use is not permitted by statutory regulation or exceeds the permitted use, you will need to obtain permission directly from the copyright holder.

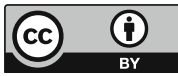

